\title{
Aspectos Fisiológicos do Mountain Biking Competitivo
}

\section{Physiological Aspects of Competitive Mountain Biking}

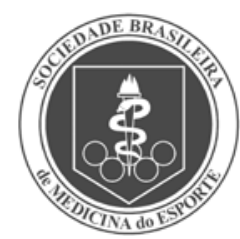

Artigo de Revisão
Ricardo Dantas de Lucas

Carlos Eduardo Polazzo Machado²

Kristopher Mendes de Souza ${ }^{1}$

Mariana Fernandes Mendes de Oliveira

Luiz Guilherme Antonacci Guglielmo'

Veronica Vleck ${ }^{3}$

Benedito Sérgio Denadai

1. Laboratório de Esforço Físico -

Universidade Federal de Santa Catarina

- Florianópolis, SC - Brasil.

2. Laboratório de Avaliação da

Performance Humana - Universidade

Estadual Paulista - Rio Claro, SP - Brasil.

3. CIPER, Faculdade de Motricidade

Humana, Universidade Tecnica de

Lisboa, Cruz Quebrada, Portugal.

Endereço para correspondência: Universidade Federal de Santa Catarina (UFSC) - Centro de Desportos -

Laboratório de Esforço Físico (LAEF)

- Bloco V - Trindade, 88040-900,

Florianópolis, SC

E-mail: ricardo@tridantas.com.br

\begin{abstract}
RESUMO
A prática do ciclismo off-road (mountain biking - MTB), cresceu muito nas últimas duas décadas, sendo incluído como esporte olímpico, nos Jogos de Atlanta em 1996, na modalidade Cross Country. Na última década, houve um aumento no número de publicações científicas que verificaram a demanda fisiológica durante competições, assim como o estudo de possíveis preditores da performance nesta modalidade. $\mathrm{O}$ objetivo deste estudo de revisão foi descrever alguns aspectos fisiológicos específicos do MTB Cross Country $\left(M^{\prime} B_{C C}\right)$ competitivo (intensidade de provas, perfil fisiológico de atletas de elite, uso de suspensões e determinantes da performance em subidas). Observa-se na literatura analisada que as provas de MTB $_{c c}$ parecem impor uma sobrecarga fisiológica maior, quando analisada através da frequência cardíaca, do que provas de ciclismo de estrada com duração semelhante. Entretanto, quando analisada pela potência de pedalada, observa-se claramente a característica intermitente da modalidade, com variações de potência durante a prova entre zero e 500W, e potência média relativamente baixa em comparação aos valores de FC encontrados. Outro fator importante levantado neste estudo são as alterações fisiológicas decorrentes do uso de suspensões nas bicicletas de $\mathrm{MTB}_{\mathrm{Cc}} \mathrm{O}$ uso deste equipamento reduz o estresse muscular provocado pelo terreno acidentado, embora pareça não afetar o gasto energético total, tanto em percurso plano como em subidas. Entretanto, é fato que o desempenho em circuitos acidentados é melhorado com o uso das suspensões. Com base nos estudos abordados nessa revisão, conclui-se que o MTB ${ }_{C c}$ enquanto modalidade competitiva apresenta uma grande variação de intensidade (avaliada através da potência), sendo esta atribuída principalmente ao tipo de terreno (irregular e com muitas aclives e declives acentuados) em que as provas de $\mathrm{MTB}_{\mathrm{CC}}$ acontecem.
\end{abstract}

Palavras-chave: ciclismo off-road, performance, competição.

\begin{abstract}
Off-road cycling (mountain biking- MTB) practice has remarkably increased over the last two decades since its debut as an Olympic summer sport in the 1996 Atlanta Games, in the Cross Country modality. The number of publications devoted to the analysis of the physiological demands and potential performance predictors in the sport has also increased over the last decade. This article provides a review of both the descriptive characteristics (such as intensity) of Cross Country MTB competition (MTBCC), as well as specific aspects related to it (such as the physiological characteristics of elite athletes, the effect of use of suspension frames and the determinants of performance on climbs). It is evident from the literature that MTBCC competitions induce greater physiological stress, when expressed in terms of $\%$ of maximal heart rate, than is observed for cycle road races of equivalent duration. Analysis of power output data clearly demonstrates the intermittent nature of this discipline- with power outputs during competition ranging between 0 and 500W and average power outputs that are relatively low as a percentage of HRmax. Another important finding is the physiological effect of the use of suspension frames in MTB. The use of such equipment reduces the muscular stress provoked by uncertain terrain without apparently influencing energy cost- either on the flat or when climbing. However, the cross country performance is improved with suspension frames. We conclude, therefore, that competitive MTBCC engenders wide variation in exercise intensity (expressed in terms of power output) - mostly as a result of the variations in terrain (i.e. irregular with many steep inclines and declines) that are a quintessential component of the sport.
\end{abstract}

Keywords: off-road cycling, performance, races. 


\section{INTRODUÇÃO}

O ciclismo off-road (mountain biking - MTB), é uma modalidade esportiva competitiva que surgiu por volta da década de 70 nos EUA. Neste período de existência, o esporte cresceu rapidamente, a ponto de ser incluído oficialmente no cenário mundial, a partir dos Jogos Olímpicos de Atlanta em 1996. De um modo geral, o MTB competitivo se difere do ciclismo tradicional (estrada e velódromo), pelo fato de ser realizado em terrenos acidentados e sem pavimentação, o que faz com que o atleta despenda mais energia para se deslocar a uma determinada velocidade ${ }^{(1,2)}$, reduzindo assim a eficiência mecânica neste esporte. Consequentemente, as bicicletas (estrada x MTB) também são bastante diferentes, sendo que as de MTB utilizam pneus mais largos e com cravos; e também são equipadas com sistemas de amortecimento (suspensão) que podem ser dianteira ou completa (dianteira e traseira).

Dentre as disputas oficiais do MTB, o Cross Country $\left(M_{T C}\right.$ ) é a forma mais popular disputada (a única modalidade olímpica), sendo realizada em circuitos fechados (estradas e trilhas de terra e pedra) contendo variação na inclinação do terreno, o que requer habilidade técnica para as descidas, e grande demanda energética para as subidas (2). A União Internacional de Ciclismo (UCI) sugere que as competições de $\mathrm{MTB}_{\mathrm{CC}}$ sejam disputadas com um tempo entre 105-135min, caracterizando assim como um evento atlético tipicamente de endurance ${ }^{(1)}$.

Contudo, embora no ciclismo tradicional muitos estudos ${ }^{(3-7)}$ têm verificado os mais diferentes aspectos fisiológicos relacionados à performance, no MTB ainda existem poucos estudos descritivos. Assim, o objetivo desta revisão é o de abordar, através da literatura existente, alguns aspectos fisiológicos relacionados à prática competitiva do $\mathrm{MTB}_{\mathrm{CC}}$, estando entre eles: 1) a característica metabólica em que ocorrem as competições de $\mathrm{MTB}_{\text {cci }}$ 2) o efeito da utilização de amortecedores sobre a performance e a demanda energética; e 3) identificar índices determinantes da performance neste esporte.

\section{Respostas fisiológicas no MTB $_{\mathrm{cc}}$}

O gasto energético do ciclismo de estrada (recreacional e competitivo) vem sendo estudado há algumas décadas ${ }^{(3,4)}$. Neste esporte, a demanda energética é dependente de três tipos de resistências ${ }^{(4)}$. Uma delas é a resistência de rolamento (contato da roda com o solo), a segunda é a resistência do ar e, por último, a resistência da gravidade. Fatores como largura do pneu, massas do ciclista e da bicicleta, velocidade de deslocamento assim como a inclinação do terreno, irão determinar o quanto cada resistência influencia no requerimento energético do ciclismo.

As condições do terreno (acidentado e montanhoso) em que as

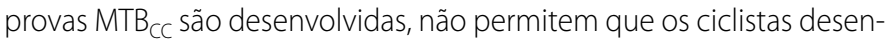
volvam velocidades similares ao ciclismo no asfalto, levando assim a um grande dispêndio de esforço contra a força da gravidade e também contra a resistência de rolamento. Associada a estes fatores observa-se também uma solicitação constante da musculatura dos braços e pernas, provenientes das contrações isométricas intensas e repetitivas, que são necessárias para absorver o impacto e as vibrações proporcionadas pelo terreno. Além disto, a baixa velocidade média em que as provas são disputadas acarreta também menor influência do drafting (técnica de pedalar atrás de outro ciclista) sobre o gasto energético.

McCole et al. ${ }^{\left({ }^{8}\right)}$ demonstraram que esta técnica diminui a resistência imposta pelo ar e, consequentemente, há uma redução no dispêndio energético. Estes autores demonstraram também que esta economia de energia aumenta exponencialmente ao aumento da velocidade de deslocamento, podendo reduzir até 40\% no gasto energético total ${ }^{(8)}$. Desta forma, no MTB, esta técnica é pouco efetiva em virtude da baixa velocidade de deslocamento em que ocorre a maioria das provas (velocidade média $<20 \mathrm{~km} . \mathrm{h}^{-1}$ ). Assim, nesta modalidade esportiva, diferentemente do ciclismo de estrada, a resistência do ar é o aspecto que apresenta menor contribuição total para o requerimento energético.

Apesar das características técnicas das provas de $\mathrm{MTB}_{\mathrm{Cc}}$ serem diferentes do ciclismo de estrada tradicional, poucos são os estudos encontrados que abordaram o requerimento energético ou a intensidade metabólica em que acontece a prática do $\mathrm{MTB}_{\mathrm{cc}}$. Berry et al. ${ }^{(9)}$, em um estudo pioneiro, verificaram o efeito da massa da bicicleta MTB e da inclinação do terreno no gasto energético e nas respostas fisiológicas. Para tal, os autores simularam em laboratório (esteira rolante) a prática do MTB, fixando um obstáculo de madeira de $3,8 \mathrm{~cm}$ de altura na esteira, de modo que a cada volta (da esteira), o obstáculo criava impacto sobre a bicicleta. Este estudo foi conduzido em três velocidades diferentes $\left(9,7,12,9\right.$ e $\left.16,2 \mathrm{~km} \cdot \mathrm{h}^{-1}\right)$ e para cada velocidade foram analisados três valores de massas de bicicletas diferentes (11,6, 12,6 e 13,6kg), assim como três inclinações diferentes (0, 2,5 e 5\%). Surpreendentemente, a massa da bicicleta não apresentou efeito significativo no consumo de oxigênio (variação de $\mathrm{VO}_{2}<1 \mathrm{ml} . \mathrm{kg} \cdot \mathrm{min}^{-1}$ ), na frequência cardíaca (FC) e na percepção subjetiva de esforço em nenhuma velocidade ou inclinação estudada ${ }^{(9)}$. Entretanto, deve-se ressaltar uma limitação, que é o fato de este estudo ter sido conduzido em laboratório.

Em relação à demanda de esforço em provas de $\mathrm{MTB}_{\mathrm{CC}}$, alguns estudos são localizados na literatura ${ }^{(1,2,10,11)}$. Impellizzeri et al. ${ }^{(1)}$ verificaram a FC durante quatro provas oficiais de $\mathrm{MTB}_{\mathrm{CC}}$ em 13 atletas de mountain bikers de elite da Itália. O tempo médio das quatro provas foi de $147 \mathrm{~min}$ ( $\pm 15 \mathrm{~min}$ ) e a $\mathrm{FC}$ média ficou próxima de $90 \%$ da $\mathrm{FC}_{\max }$ o que correspondeu a $84 \%$ do consumo máximo de oxigênio $\left(\mathrm{VO}_{2 \max }\right)$ dos atletas ${ }^{(1)}$. Este $\% F C_{\text {max }}$ representa a intensidade do limiar anaeróbio medido em laboratório, no qual supostamente os atletas deveriam entrar em exaustão entre 40min e 1h, embora as provas tenham apresentado duração superior a $2 \mathrm{~h}$.

A discrepância entre \%FC $\max$ e duração das provas do estudo anterior foi confirmada por mais dois estudos. Prins et al. ${ }^{(10)}$ encontraram valor médio de $88,5 \%$ da FC $_{\max }$ (em uma prova com duração média de 106min) para oito mountain bikers de nível regional e nacional da África do Sul. Stapelfeldt et al.(2) mensuraram a FC e a potência gerada em 11 mountain bikers de elite da Alemanha, durante 15 provas oficiais de $\mathrm{MTB}_{\mathrm{CC}}$. Os resultados encontrados nesse estudo corroboraram aqueles encontrados no estudo de Impellizzeri et al. ${ }^{(1)}$ com relação à $F C$, visto que os valores foram similares $\left(177 \pm 6 \mathrm{bpm}=91 \%\right.$ da $\left.\mathrm{FC}_{\max }\right)$ em provas que duraram em média 128min ( $\pm 17 \mathrm{~min}$ ). Além disso, os autores verificaram uma baixa variação intraindividual da FC durante as provas analisadas (2). Entretanto, houve dissociação entre a FC e a potência, quando considerados como indicadores de intensidade de esforço. A FC permaneceu elevada durante todo o percurso, enquanto que a potência variou entre 0 a $500 \mathrm{~W}$, respectivamente, para as descidas e subidas. A potência média foi de 246W nas 15 corridas, o que correspondeu a aproximadamente $67 \%$ da potência máxima $\left(P_{\max }\right)$ obtida no teste incremental (vs. 91\% da $\left.\mathrm{FC}_{\max }\right)^{(2)}$.

Gregory et al. ${ }^{(11)}$ também verificaram o perfil da potência durante uma simulação de corrida $\mathrm{MTB}_{\mathrm{CC}} \mathrm{com} 15,5 \mathrm{~km}$ de distância e encontram valor médio de potência de $315 \mathrm{~W}$, que representou $85,5 \%$ da $P_{\max }$ do teste incremental de laboratório. Novamente, o valor médio da FC durante esta simulação de corrida, que teve duração média de $61 \mathrm{~min}$, foi de $91 \%$ da $F C_{\max }$. Estes autores ainda verificaram os valores médios da potência e FC em diferentes trechos do percurso, encontrando valores extremos de $420 \mathrm{~W}$ (115\% da $\mathrm{P}_{\max }$ ) em subidas mais inclinadas e $20 \mathrm{~W}$ $\left(17 \%\right.$ da $\left.P_{\max }\right)$ nas descidas mais acentuadas ${ }^{(11)}$. Estes dados descritivos 
de potência em corridas oficiais de $\mathrm{MTB}_{\mathrm{CC}}$ mostraram uma grande variação de valores durante a prova, o que parece traduzir de forma mais consistente a característica fisiológica intermitente deste esporte.

Padilla et al. ${ }^{(7)}$ estudaram duas provas curtas de contrarrelógio individual (CR) no ciclismo de estrada (duração média de 10 e 40min) e encontraram valores médios de FC correspondentes a 89 e $85 \%$ da $F C_{\text {max }}$ respectivamente. Desta forma, se analisarmos estes valores de provas CR em estrada, com os reportados por Impellizzeri et al. ${ }^{(1)} \mathrm{e}$ Stapelfeldt et al. ${ }^{(2)}$ para provas de $\mathrm{MTB}_{\mathrm{CC}}$ observamos valores de $\% \mathrm{FC}_{\max }$ muito semelhantes; no entanto, com durações médias de provas bem diferentes $\left(\mathrm{MTB}_{\mathrm{CC}} \sim 120-150 \mathrm{~min}\right.$ vs. CR 10-40min).

A parir destes estudos, pode-se inferir que a FC não é um indicador de esforço confiável para estimar a intensidade metabólica em provas de $\mathrm{MTB}_{\mathrm{cc}}$ já que este índice sofre influência de pelo menos dois fatores frequentes neste esporte: 1) repetidas contrações isométricas para controlar a bicicleta em terrenos acidentados, principalmente de membro superior; e 2) grande descarga adrenérgica proveniente do estresse emocional durante descidas com dificuldade técnica.

Ao analisar todas as intensidades de esforço durante as provas de $\mathrm{MTB}_{\mathrm{CC}}$ os dados confirmam a dissociação entre FC e potência. Impellizzeri et al. ${ }^{(1)}$ determinaram em laboratório a $\mathrm{FC}$, a potência e o $\mathrm{VO}_{2}$ correspondentes ao limiar de lactato (LL), limiar anaeróbio (OBLA) e $\mathrm{VO}_{2 \max }$ de cada atleta. Posteriormente, somente a $\mathrm{FC}$ foi monitorada em quatro provas oficiais da $\mathrm{UCl}$ e estimou-se o percentual do tempo em que os atletas permaneceram em cada zona de intensidade (fácil, moderada e difícil), como proposto por Gilman ${ }^{(12)}$. A zona fácil representava intensidades de exercício situadas abaixo da FC do LL; a zona moderada, intensidades de exercício encontradas entre a FC correspondente ao LL e ao OBLA; e a zona difícil, intensidades de exercício situadas acima da FC do OBLA(12).

Stapelfeldt et al. ${ }^{(2)}$ determinaram também em laboratório as seguintes zonas e as potências das intensidades (figura 1): limiar aeróbio (LAer) - (menor razão entre $\mathrm{VO}_{2}$-lactato); limiar anaeróbio individual (IAT); e potência correspondente ao $\mathrm{VO}_{2 \max }\left(P_{\max }\right)$. A medição da potência durante as provas foi realizada por meio do sistema conhecido como SRM (Schberer Rad Messtechnik), que detecta a potência aplicada ao pé de vela da bicicleta de cada atleta, durante a situação de competição. Houve discordâncias ao comparar o tempo sustentado em cada uma das zonas de intensidade entre os estudos de Impellizzeri et al.(1) e Stapelfeldt et al. ${ }^{(2)}$, como descrito na figura 1.

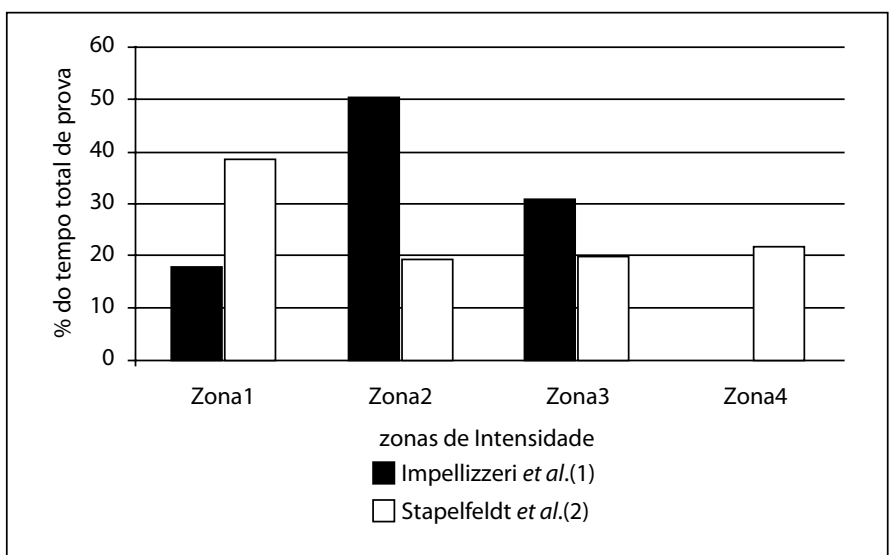

Figura 1. Representação da \% do tempo de prova gasto (valores médios) em cada zona de intensidade, analisada em dois estudos diferentes. Zona 1 (abaixo do LL para Impellizzeri et al. (1) e abaixo do LAer para Stapelfeldt et al. ${ }^{(2)}$ ); Zona 2 (entre LL e OBLA para Impellizzeri et al. ${ }^{(1)}$ e entre LAer e IAT para Stapelfeldt et al. ${ }^{(2)}$ ); Zona 3 (acima do OBLA para Impellizzeri et al. ${ }^{(1)}$ e entre IAT e $P_{\max }$ para Stapelfeldt et al.(2)); Zona 4 (acima da $\left.P_{\max }\right)$. É importante ressaltar que Impellizzeri et al. ${ }^{(1)}$ se baseou na FC e Stapelfeldt et al. ${ }^{(2)}$ se baseou na potência de cada índice para determinar cada zona. Impellizzeri et al. ${ }^{(1)}, N=8$ observações; Stapelfeldt et al. ${ }^{(2)}, N=36$ observações.
Impellizzeri et al. ${ }^{(1)}$, utilizando a FC para estimar o tempo em cada uma das três zonas de intensidade, verificaram valores médios de 18, 51 e $31 \%$ do tempo total das provas, respectivamente para as zonas fácil, moderada e difícil (figura 1). O tempo absoluto médio gasto na zona difícil (> OBLA) foi de 44min, sendo este um valor maior do que o reportado por Padilla et al. ${ }^{(13)}$ em etapas de montanha na Volta da França (16min na zona difícil). Considerando todos estes dados, os autores sugerem que competições de $\mathrm{MTB}_{\mathrm{Cc}}$ exigem a utilização de um elevado percentual da potência aeróbia máxima, por períodos de tempo prolongado. Entretanto, considerando os fatores discutidos acima, estes percentuais parecem não refletir mais uma vez a real intensidade metabólica das provas. Desta forma, o estudo de Stapelfeldt et al. ${ }^{(2)}$ parece ser mais apropriado para analisar a distribuição de diferentes zonas de intensidade, embora neste estudo tenham sido utilizados critérios um pouco diferentes para dividir cada zona (figura1).

Com os resultados do estudo de Stapelfeldt et al.(2), observa-se que em aproximadamente $40 \%$ do tempo de prova, os atletas geravam potência inferior ao LL, enquanto em outros $20 \%$ do tempo total foi gerado potência superior à $\mathrm{P}_{\text {max }}$ (intensidade supramáxima). Estes dados enfatizam a grande variação de potência encontrada nesta modalidade, o que pode sugerir especificidades na prescrição de treinamento de atletas de MTB $_{c c}$.

Outro fato bastante interessante encontrado no estudo de Impellizzeri et al. ${ }^{(1)}$ foi em relação à estratégia de competição adotada pelos mountain bikers. Os autores observaram que a FC diminuiu durante o desenrolar da corrida e que o maior valor de FC (191 \pm 6bpm) foi atingido logo após a largada. Possivelmente, esta estratégia está relacionada ao fato de se tentar obter um melhor posicionamento logo no início da prova, já que muitas vezes os atletas encontram passagens estreitas e trilhas individuais (single track) neste tipo de prova. Outro aspecto que confirma esta elevada intensidade inicial é a redução da velocidade média verificada volta após volta nas provas estudadas. Desta forma, os autores supracitados sugerem que no treinamento desta modalidade deve haver uma tentativa de adaptação semelhante a esta. Contudo, mais estudos são necessários para melhor compreender o quanto esta estratégia auxilia no desempenho final.

\section{Características fisiológicas de mountain bikers competitivos}

Nos últimos anos, alguns estudos vêm tentando comparar as respostas fisiológicas relacionadas ao desempenho, entre ciclistas de estrada e mountain bikers. Wilber et al. ${ }^{(14)}$ estudaram 20 ciclistas de estrada (10 homens e 10 mulheres) e 20 mountain bikers (10 homens e 10 mulheres), ambos de nível internacional e que representavam a equipe norte-americana de ciclismo e MTB, respectivamente. Os autores avaliaram alguns índices máximos (relacionados ao $\mathrm{VO}_{2 \max }$ ) e submáximos (relacionados ao OBLA) durante um teste incremental de laboratório. Os autores concluíram que os mountain bikers de elite apresentam um perfil fisiológico $\left(\mathrm{VO}_{2 \text { maxi }} ; \mathrm{P}_{\text {maxi }} ; \mathrm{OBLA}\right)$ similar aos ciclistas de estrada de elite.

Mais recentemente, Lee et al. ${ }^{(15)}$ também estudaram alguns parâmetros fisiológicos entre mountain bikers e ciclistas de estrada profissionais. O grupo de mountain bikers estudado apresentou uma menor massa corporal e percentual de gordura que o grupo de ciclistas de estrada. Os valores médios de $\mathrm{VO}_{2 \max }\left(78,3 \pm 4,4\right.$ vs. $\left.73,0 \pm 3,4 \mathrm{ml} . \mathrm{kg} \cdot \mathrm{min}^{-1}\right), \mathrm{P}_{\max }$ relativa $\left(6,3 \pm 0,5\right.$ vs. $\left.5,8 \pm 0,3 W \cdot \mathrm{kg}^{-1}\right), \mathrm{OBLA}(5,2 \pm 0,6$ vs. 4,7 $\pm 0,3 \mathrm{~W}$. $\left.\mathrm{kg}^{-1}\right)$ e potência média em um teste $C R$ de 30 min $(5,5 \pm 0,5$ vs. 4,9 \pm $0,3 \mathrm{~W} . \mathrm{kg}^{-1}$ ) foram significantemente maiores nos mountain bikers ${ }^{(15)}$. Os valores dos mountain bikers acima citados são similares aos valores reportados por Padilla et al. ${ }^{(13)}$ para ciclistas de estrada profissionais, especialistas em subidas. Estes resultados mostram que, para uma boa performance no $\mathrm{MTB}_{\mathrm{cc}}$ é importante também a produção de uma alta potência aeróbia por unidade de massa corporal. 
Costa et al. ${ }^{(16)}$ reportaram valores de índices fisiológicos em mountain bikers brasileiros, com diferentes níveis de performance (regional, nacional e internacional) e idade. Os valores médios desta amostra aparentemente heterogênea $(N=29)$ para $P_{\max }$ relativa foi de $4,7 \pm$ $0,4 \mathrm{~W} \mathrm{~kg}^{-1}$, o que é um valor similar à potência do OBLA para a amostra do estudo de Lee et al. ${ }^{(15)}$. Interessantemente, embora a amostra do estudo de Costa et al. ${ }^{(16)}$ pareça ser heterogênea, verifica-se um baixo coeficiente de variação (CV) para todos os índices estudados ( 10\%). Os valores do atleta brasileiro de MTB $_{C c}$, Rubens Donizete Valeriano (medalha de prata nos Jogos Pan-Americanos em 2007 e 210 lugar nos Jogos Olímpicos de Pequim - 2008), recentemente verificado em nosso laboratório $\left(P_{\max }=5,7 \mathrm{~W} \cdot \mathrm{kg}^{-1} ; \mathrm{OBLA}=4,5 \mathrm{~W} \cdot \mathrm{kg}^{-1} ; \mathrm{LL}=4,1 \mathrm{~W} \cdot \mathrm{kg}^{-1}\right)$, são compatíveis com os atletas de nível internacional.

Baron $^{(17)}$ realizou um estudo com intuito de verificar as características aeróbias e anaeróbias de 25 mountain bikers austríacos de elite com experiência internacional. Neste estudo, foi realizado um teste incremental máximo para determinar os índices aeróbios $\left(P_{\text {maxAer }} \mathrm{e}\right.$ OBLA) e também um teste isocinético de 10seg para determinar a potência anaeróbia média e máxima $\left(P_{\operatorname{maxAn}}\right)$ em diferentes cadências de pedalada (de 50 a 140rpm). Foi encontrada a maior potência média nesse estudo na cadência de 100rpm, considerando esta a cadência ótima para esforços em torno de 10 seg $^{(17)}$. Outro aspecto interessante foi a relação obtida entre a $P_{\operatorname{maxAer}}$ e a $P_{\max A n}$ sendo denominada no estudo como índice de potência $\left(P_{\text {maxAer }} / P_{\text {maxAn }} \times 100\right)^{(17)}$. Os mountain bikers apresentaram um valor médio deste índice de 38\%, contra 32\% de um grupo controle (estudantes) ${ }^{(17)}$.

Este índice demonstra uma proporção entre a potência aeróbia e anaeróbia, sendo diretamente dependente da especialização nos treinamentos e competições ${ }^{(17)}$. Infelizmente, ainda não existem estudos utilizando este índice com ciclistas para realizar uma comparação destes resultados ou mesmo para estabelecer padrões de referência. Além disso, seria interessante utilizar estudos correlacionais para detectar o poder de predição deste índice com a performance do MTB $\mathrm{CC}_{\mathrm{C}}$ assim como a performance em subidas.

\section{Fatores determinantes da performance no $\mathrm{MTB}_{\mathrm{cc}}$}

Em função de o perfil fisiológico das provas de $\operatorname{MTB}_{C C}(1,2,11)$ ser diferente do ciclismo tradicional, alguns estudos recentes têm verificado o poder de predição dos principais índices fisiológicos obtidos em testes ergométricos de laboratório sobre a performance em provas $\mathrm{MTB}_{\mathrm{CC}}(10,18,19)$.

Impellizzeri et al. ${ }^{(18)}$ verificaram a correlação entre as variáveis máximas e submáximas (através de parâmetros de lactato sanguíneo) obtidas em um teste incremental (incrementos de 40W e estágios de $4 \mathrm{~min}$ ), com uma prova oficial de $\mathrm{MTB}_{\mathrm{Cc}}$ com $31 \mathrm{~km}$ de distância e $1.260 \mathrm{~m}$ de altitude acumulada. Ao todo, foram estudados 13 mountain bikers do gênero masculino, de nível nacional e internacional. Neste estudo, foram encontradas correlações significantes entre o $\mathrm{VO}_{2 \text { max }} \mathrm{P}_{\text {max }}, \mathrm{OBLA}$ e $L L$ expressos de forma absoluta e relativa à massa corporal, com o tempo de prova e também com o ranking final da prova (r entre 0,62 e $0,96)$, sendo que o maior preditor foi o OBLA relativo à massa corporal ou à massa corporal ${ }^{-0,79}$. Esta alta correlação obtida entre as variáveis de laboratório, tanto de potência quanto de capacidade aeróbia, pode ser justificada em parte pela "heterogeneidade" do grupo estudado (CV $=7,5 \%)$.

Em outro estudo de Impellizzeri et al. ${ }^{(19)}$, foram estudados 12 mountain bikers italianos do gênero masculino, todos com experiência em provas internacionais e com nível de performance homogênea (CV = $1,5 \%)$. Os autores verificaram a correlação entre as variáveis máximas e também submáximas obtidas através de variáveis ventilatórias (limiar ventilatório - LV1 - e limiar de compensação respiratória ou segundo limiar ventilatório - LV2) com uma prova de nível internacional com distância de 33,6km e altitude acumulada de $1.362 \mathrm{~m}$. Contraditoriamente ao estudo anterior, as únicas variáveis que apresentaram correlação moderada com o tempo de prova foram o $\mathrm{VO}_{2}$ e a potência (expressos de forma relativa à massa corporal) no LV2 (respectivamente, $r=0,66$ e 0,63), explicando em 40\% a variação da performance da prova. Estes valores relativamente baixos de correlação entre a performance e as variáveis fisiológicas em atletas de elite mostram que as provas de $\mathrm{MTB}_{\mathrm{CC}}$ podem sofrer influência de outros fatores fisiológicos (capacidade e potência anaeróbia), assim como da habilidade técnica para pilotar a bicicleta em terrenos acidentados.

Em outro estudo, Prins et al. ${ }^{(10)}$ verificaram correlações significativas somente entre a $P_{\max }$ relativa $(r=0,83)$ e a performance em uma prova de $32 \mathrm{~km}$, em um grupo de oito mountain bikers da África do Sul (CV de performance $=6,3 \%$ ). Interessantemente, quando estes mesmos atletas refizeram o mesmo percurso da prova em forma de CR (tomada de tempo individual), o CV de performance foi de 4,3\%, e observaram-se correlações novamente com a $P_{\max }(r=0,83)$ e também com o OBLA, ambos os índices relativos à massa corporal $(r=0,74)^{(10)}$. Desta forma, estes resultados sugerem que o fato de as provas oficiais serem realizadas com largada simultânea de todos os competidores (ao invés de CR) pode interferir nas correlações com as variáveis fisiológicas submáximas obtidas em laboratório, em função das estratégias de largada e consequentemente possíveis dificuldades de ultrapassagens durante as provas oficiais.

Adicionalmente, Prins et al. ${ }^{(10)}$ propuseram um teste específico de laboratório para o $\mathrm{MTB}_{\mathrm{CC}}$ que consistiu em um CR de $1 \mathrm{~km}$ em laboratório $\left(C_{1 \mathrm{~km}}\right)$ realizado em três situações: $\left.1^{\underline{a}}\right)$ condição de repoubso; $\left.2^{\underline{a}}\right)$ após um teste de laboratório de carga variável simulando uma volta do percurso da prova ( 26min); e 3âa) após a simulação de duas voltas do percurso da prova. Não foram encontradas correlações entre as três situações do $C R_{1 \mathrm{~km}}$ com as performances no percurso da prova, demonstrando que o teste específico proposto para avaliar mountain bikers não apresentou validade para tal fim.

Assim, de acordo com os resultados dos estudos apresentados, observou-se que a performance em provas oficiais de MTB $_{c c}$, parece ser melhor explicada por variáveis submáximas (referentes à capacidade aeróbia) sempre normalizadas à massa corporal do ciclista, sendo assim semelhante aos ciclistas de estrada especialistas em subida.

\section{Fatores determinantes da performance em subidas}

Os tipos de terreno têm uma importância grande para o desempenho final em provas de MTB, já que quase sempre os circuitos CC são realizados em locais com muitas subidas e descidas (a UCl aconselha que os circuitos oficiais tenham ascensão acumulada em torno de $1.500 \mathrm{~m})^{(1,2)}$.

A performance e a demanda fisiológica do ciclismo em subidas têm sido verificadas por diversos estudos nestes últimos anos, tanto no ciclismo tradicional ${ }^{(8,13,20-22)}$, quanto no $\mathrm{MTB}_{C \mathrm{C}}{ }^{(2,23,24)}$. Um fator conhecido, que influencia negativamente a performance em subidas, é a massa corporal(6,20,24). Quando o ciclismo é praticado em subidas, ocorre uma maior ação da força gravitacional e, em função da velocidade de deslocamento ser menor, ocorre também uma redução da ação da resistência do ar. Desta forma, uma grande porção da energia despendida é utilizada para vencer a força da gravidade, sendo esta influenciada diretamente pela massa corporal do ciclista e a massa bicicleta. Segundo Swain e Wilcox ${ }^{(20)}$, cerca de 10 a 20\% da variação da performance em ciclistas de elite, em condições de subida, é explicada por diferenças na massa corporal, visto que os ciclistas mais leves são os que apresentam maior performance.

Outros estudos ${ }^{(20-22)}$ também têm demonstrado que, em ciclistas profissionais, a $P_{\max }$ e o OBLA (ambos em W. $\mathrm{kg}^{-1}$ ) são índices que 
predizem a performance em subidas entre um e $12 \mathrm{~km}$ de extensão e gradientes de inclinação (distância percorrida/diferença de altitude) de seis a 12\%. O metabolismo anaeróbio também parece contribuir de forma significativa para o rendimento em subidas realizadas com bicicletas tradicionais, visto que Davison et al.(21) encontraram uma alta correlação entre a potência média relativa gerada no teste Wingate (30seg) e o tempo registrado em subidas de $1 \mathrm{~km}(r=-0,92)$ e $6 \mathrm{~km}(r=-0,90)$, simuladas em uma esteira.

Aparentemente, o tipo de terreno (asfalto ou terra) pode promover diferenças principalmente nas técnicas para a estabilização do centro de massa e estratégias de mudanças de marchas durante subidas íngremes, já que normalmente existe uma constante redução da aderência dos pneus com a terra (ou pedras). Machado et al. ${ }^{(23)}$ realizaram um estudo com o objetivo de verificar a influência de variáveis antropométricas e metabólicas (aeróbias e anaeróbias) sobre a performance do MTB em subida. Para tal, foi estudada uma subida de terra/pedras de $1,4 \mathrm{~km}$ de extensão e gradiente de inclinação médio de 15\%. A velocidade média de subida registrada foi de $8,7 \mathrm{~km} \cdot \mathrm{h}^{-1}$. Os valores obtidos em um teste incremental de laboratório ( $P_{\max }$ e OBLA), um teste de Wingate, juntamente com algumas variáveis antropométricas (massa total, índice de massa corpórea, percentuais de massa gorda e magra) foram correlacionados com o tempo registrado na subida. O percentual de gordura corporal (\%GC) dos atletas estudados (6,9 \pm $3,0 \% ; N=9)$ foi a variável que obteve melhor correlação $(r=0,72)$ com a performance ${ }^{(23)}$, explicando aproximadamente $52 \%$ da variação de desempenho desta subida. Em relação às variáveis fisiológicas, não foi encontrada correlação significante entre a potência anaeróbia média $\left(W \mathrm{~kg}^{-1}\right)$ medida no teste de Wingate com o desempenho, mesmo esta atingindo altos valores de lactato final (média de $14 \mathrm{mmol} . \mathrm{L}^{-1}$ ), o que sugere uma grande solicitação do metabolismo glicolítico anaeróbio ${ }^{(23)}$. Entretanto, foi encontrada uma correlação significante com a $P_{\max }(r=$ $-0,72)$ e o OBLA $(r=-0,87)$, ambos em W. $\mathrm{kg}^{-1}$, concordando com os resultados obtidos por Padilla et al. ${ }^{(13)}$ para o ciclismo tradicional. Além disso, Lucia et al.(22) relataram que a performance em subida além de ser dependente de uma alta $P_{\max }$ (aeróbio) também depende de uma ótima capacidade de tamponamento do sistema anaeróbio e de um maior padrão de recrutamento de unidades motoras.

Dessa forma, com base nos estudos apresentados, pode-se perceber que, dependendo da duração das subidas e também do gradiente de inclinação, os sistemas energéticos (aeróbio e anaeróbio) podem contribuir em diferentes proporções, no entanto, sempre relacionados à massa corporal. A princípio, podemos afirmar que subidas que apresentam uma duração superior a 8min, sofrem uma grande contribuição do metabolismo aeróbio, mesmo com gradiente de inclinação superior a $10 \%$

\section{Efeito da utilização de bicicletas com suspensão sobre a performance e variáveis fisiológicas}

O uso do sistema de absorção de impacto (suspensão) utilizado nas bicicletas de MTB tem sido amplamente difundido e melhorado em termos de eficiência e leveza. Hoje em dia, existem dois tipos de bicicletas equipadas com este acessório. Existem as bicicletas com suspensão dianteira, que substitui o garfo rígido utilizado nas bicicletas comuns, e as bicicletas equipadas com uma suspensão dianteira e uma suspensão traseira (completa), que permite a absorção de impactos gerados sobre o quadro inteiro da bicicleta. Este segundo sistema de suspensão é muito utilizado em provas de descida conhecidas como downhill, embora também tenha sido utilizado em provas de $\mathrm{MTB}_{\mathrm{Cc}}$.

Seifert et al. ${ }^{(25)}$ demonstraram experimentalmente que o uso de suspensão dianteira na bicicleta reduz significantemente os níveis de creatina quinase plasmática (CK) quando medida 24 horas após CR de uma hora, em um percurso acidentado, comparado ao garfo rígido. Em um outro estudo, Nishii et al. (26) compararam os níveis de CK entre os dois tipos de suspensões (dianteira vs. completa), 24 horas após CR de 30min em um circuito de $\mathrm{MTB}_{\mathrm{Cc}}$ com 950m de extensão. Os autores encontraram uma menor concentração de CK quando foi utilizada a suspensão completa(26). Desta forma, estes dois estudos ${ }^{(25,26)}$ sugerem que a utilização das suspensões gera um menor estresse muscular decorrente de contrações isométricas e eventualmente excêntricas, comuns na prática do MTB.

Para testar se as suspensões utilizadas nas bicicletas de MTB poderiam influenciar a demanda energética durante subidas, MacRae et al. ${ }^{(27)}$ compararam os dois tipos de suspensão. A principal hipótese era de que a suspensão completa requereria um maior gasto energético em função do maior peso deste equipamento. No entanto, não foram encontradas diferenças nas variáveis fisiológicas avaliadas $\left(\mathrm{VO}_{2}\right.$ FC, lactato) em uma subida de 1,4km com uma taxa de inclinação de $11 \%$, sugerindo, assim, que a suspensão completa não apresenta efeito na demanda energética em subidas, como normalmente é afirmado em outros estudos. Atualmente, com a evolução dos materiais, até mesmo o peso do sistema completo é equivalente a muitas bicicletas equipadas somente com a suspensão dianteira, como verificado no estudo conduzido por Nishii et al. ${ }^{(26)}$.

Em um estudo mais amplo, Seifert et al. ${ }^{(25)}$ estudaram o efeito das suspensões sobre respostas fisiológicas e também sobre a performance no MTB. Estes autores não encontraram diferenças nas performances de subida $(0,76 \mathrm{~km})$ e descida $(0,76 \mathrm{~km})$ entre os três tipos de sistema utilizados normalmente (garfo rígido, suspensão dianteira ou suspensão completa). Entretanto, em um circuito de $\operatorname{MTB}_{C C}(10,44 \mathrm{~km})$, o uso da suspensão dianteira gerou uma melhora de 80 seg sobre os outros dois sistemas ${ }^{(25)}$. Em relação ao requerimento energético, não foi encontrada diferença entre os três tipos de sistema, embora os atletas tenham relatado uma menor percepção subjetiva de esforço e também maior conforto, quando a suspensão completa foi utilizada.

Em outro estudo, Nielens e Lejeune ${ }^{(28)}$ confirmaram estas evidências experimentais não encontrando também diferenças no requerimento energético medido em laboratório, entre os dois tipos de amortecimento. $O$ estudo mais recente realizado para verificar o efeito dos dois tipos de suspensões sobre a performance foi o de Nishii et al. ${ }^{(26)}$. Neste estudo, foram avaliados oito mountain bikers japoneses de elite $\left(\mathrm{VO}_{2 \max }\right.$ $\left.=67,8 \pm 5,8 \mathrm{ml} \cdot \mathrm{kg} \cdot \mathrm{min}^{-1}\right)$, que realizaram duas performances de $30 \mathrm{~min}$ em um circuito de $\mathrm{MTB}_{\subset \mathrm{cc}}$ com $950 \mathrm{~m}$ de extensão(26). Em cada performance, foi utilizado um tipo de suspensão (dianteiro vs. completo), sendo que a massa total das bicicletas equipadas com estes dois sistemas foi semelhante (10,4 vs. 10,8kg), diferentemente dos estudos anteriormente citados. Neste estudo, Nishii et al. ${ }^{(26)}$ verificaram uma velocidade média $5,2 \%$ superior $\left(24,1\right.$ vs. 22,9km.h $\left.\mathrm{h}^{-1}\right)$ e consequentemente maior distância percorrida $(12,1$ vs. $11,5 \mathrm{~km})$ nos $30 \mathrm{~min}$ CR, quando foi utilizado o sistema de suspensão completa. Interessantemente, a cadência média também foi maior com este tipo de suspensão(26). As outras variáveis analisadas (FC, potência, lactato) não foram significantemente diferentes entre as duas condições. O melhor desempenho verificado com o sistema completo de suspensão verificado neste estudo(26), evidencia mais uma vez a melhor absorção de impacto dos sistemas modernos de suspensão, permitindo assim um melhor desempenho em provas de $\mathrm{MTB}_{\mathrm{Cc}}$ quando o efeito da massa das bicicletas é isolado.

\section{CONCLUSÃO}

Com base nos estudos existentes, é possível concluir que o MTB $\mathrm{Cc}_{\mathrm{C}}$ disputado nas competições oficiais com valores médios relativamente estáveis em torno de $90 \%$ da $\mathrm{FC}_{\text {max }}$ por um período de esforço próximo a 120min. Entretanto, este alto valor relativo da FC parece não refletir a 
real demanda de esforço avaliada através do perfil da potência (W) gerada nas competições. Através do perfil da potência é possível verificar uma característica altamente intermitente nas provas, com variações de zero (descidas) a 500W (subidas curtas) em mountain bikers de elite. Em relação aos índices fisiológicos comumente utilizados em laboratório para avaliar a potência e a capacidade aeróbia, são encontradas discrepâncias na literatura, quando se analisa as correlações com a performance das provas de MTB $_{\text {cc. }}$. Entretanto, a maioria das evidências aponta para uma maior correlação das variáveis submáximas (referentes à capacidade aeróbia) sempre normalizadas à massa corporal do ciclista, apresentando assim características fisiológicas semelhantes aos ciclistas de estrada especialistas em subida. Quanto ao uso de amor- tecedores, estes apresentam uma vantagem significativa na prática do MTB em relação ao uso do garfo/quadro rígido. Contudo, com a tecnologia atual, na qual as bicicletas com sistema completo de suspensão (dianteiro e traseiro) têm massa total equivalente às bicicletas com sistema dianteiro somente, o ganho de performance associado à redução do estresse muscular é favorável às bicicletas equipadas com o sistema completo.

Todos os autores declararam não haver qualquer potencial conflito de interesses referente a este artigo.

\section{REFERÊNCIAS}

1. Impellizzeri F, Sassi A, Rodriguez-Alonso M, Mognoni P, Marcora S. Exercise intensity during off-road cycling competitions. Med Sci Sports Exerc 2002;34:1808-13.

2. Stapelfeldt B, Schwirtz A, Schumacher YO, Hillebrecht M. Workload demands in mountain bike racing. Int J Sports Med 2004;25:294-00.

3. Davies CT. Effect of air resistance on the metabolic cost and performance of cycling. Eur J Appl Physiol 1980;45:245-54.

4. di Prampero PE, Cortili G, Mognoni P, Saibene F. Equation of motion of a cyclist. J Appl Physiol 1979;47:201-6.

5. Lucia A, Hoyos J, Carvajal A, Chicharro JL. Heart rate response to professional road cycling: the Tour of France. Int J Sports Med 1999;20:167-72.

6. Swain, DP. The influence of body mass in endurance bicycling. Med Sci Sports Exerc 1994;26:58-63.

7. Padilla S, Mujika I, Orbañanos J, Angulo F. Exercise intensity during competition time trials in professional road cyclists. Med Sci Sports Exerc 2000;32:850-6.

8. McCole SD, Claney K, Conte JC, Anderson R, Hagberg JM. Energy expenditure during bicycling. J Appl Physiol 1990;68:748-53.

9. Berry MJ, Koves TR, Benedetto JJ. The influence of speed, grade and mass during simulated off road bicycling. Appl. Ergonomics 2000;31:531-6

10. Prins $L$, Terblanche $E$, Myburgh KH. Field and laboratory correlates of performance in competitive cross-country mountain bikers. J Sports Sci 2007;25:927-35

11. Gregory J, Johns DP, Walls JT. Relative vs. absolute physiological measures as predictors of mountain bike cross-country race performance J Strength Cond Res 2007;21:17-22.

12. Gilman MB. The use of heart rate to monitor the intensity of endurance training. Sports Med 1996;21:73-9.

13. Padilla S, Mujika I, Cuesta G, Goiriena J. Level ground and uphill cycling ability in professional road cycling. Med Sci Sports Exerc 1999, 31:878-85.

14. Wilber RL, Zawadzki KM, Kearney JT, Shannon MP, Disalvo D. Physiological profile of elite off-road and road cyclists. Med Sci Sports Exerc 1997;29:1090-4.

15. Lee H, Martin DT, Anson JM, Grundy D, Hahn AG. Physiological characteristics of successful mountain bikers and professional road cyclists. J Sports Sci 2002;20:1001-8.

16. Costa VP, Nakamura, FY, De-Oliveira, FR. Aspectos fisiológicos e de treinamento de mountain bikers brasileiros. Rev Ed Fis 2007;136:5-11.

17. Baron R. Aerobic and anaerobic power characteristics of off-road cyclists. Med Sci Sports Exerc 2001;33:1387-93.

18. Impellizzeri FM, Rampinini E, Sassi A, Mognoni P, Marcora S. Physiological correlates to off-road cycling performance. J Sports Sci 2005;23:41-7.

19. Impellizzeri FM, Marcora SM, Rampinini E, Mognoni P, Sassi A Correlations between physiological variables and performance in high level cross country off road cyclists. Br J Sports Med 2005;39:747-51.

20. Swain DP, Wilcox JP. Effects of cadence on the economy of uphill cycling. Med Sci Sports Exerc 1992;24:1123-7.

21. Davison RC, Swan D, Coleman D, Bird S. Correlates of simulated hill climb cycling performance. J Sports Sci 2000;18:105-110

22. Lucia A, Joyos $\mathrm{H}$, Chicarro J. Physiological response to professional road cycling: climbers vs. time trialists. Int J Sports Med 2000;21:505-12.

23. Machado CEP, Caputo F, de Lucas RD, Denadai BS. Fatores fisiológicos e antropométricos associados com a performance em subida no ciclismo off road. Rev Bras Cien Mov 2002;10:35- 40.

24. Impellizzeri FM, Ebert T, Sassi A, Menaspa P, Rampinini E, Martin DT. Level groud and uphill cycling ability in elite female mountain bikers and road cyclists. Eur J Appl Physiol 2008;102:335-41.

25. Seifert JG, Luetkemeier MJ, Spencer MK, Miller D, Burke ER. The effects of mountain bike suspension systems on energy expenditure, physical exertion, and time trial performance during mountain bicycling. Int J Sports Med 1997;18:197-00.

26. Nishii T, Umemura Y, Kitagawa K. Full suspension mountain bike improves off-road cycling performance J Sports Med Phys Fitness 2004;44:356-60

27. MacRae HS-H, Hise KJ, Allen PJ. Effects of front and dual suspension mountain bike systems on uphil cycling performance. Med Sci Sports Exerc 2000;32:1276-80

28. Nielens $\mathrm{H}$, Lejeune TM. Energy cost of riding bicycles with shock absorption systems on a flat surface Int J Sports Med 2001;22:400-4 